\title{
Two defence applications involving discrete valued optimal control
}

\author{
V. Rehbock* L. Caccetta ${ }^{\dagger}$
}

(Received 2 January 2002; revised 13 February 2002)

\begin{abstract}
We present two examples of optimal discrete valued control problems which arise in defence applications. The first one involves the management of batteries in a submarine. In the second example, one wishes to determine an optimal transit path for a submarine through a field of sonar sensors, subject to a total time constraint. A recently developed numerical solution technique for discrete valued optimal control problems is used to solve both of these problems. We present numerical results for each example.
\end{abstract}

${ }^{*}$ Department of Mathematics and Statistics, Curtin University of Technology, GPO Box U 1987, Perth 6845, Australia.

mailto:rehbock@maths . curtin.edu.au

†as above. mailto: caccetta@maths. curtin.edu.au

${ }^{0}$ See http: //anziamj . austms .org.au/V44/E046 for this article, (C) Austral. Mathematical Soc. 2002. Published July 9, 2002. ISSN 1446-8735 


\section{Contents}

1 Introduction

E34

2 Problem Formulation and Solution Strategy

E35

3 The Optimal Battery Recharge Problem

E39

3.1 The Optimal Control Model . . . . . . . . . . . E40

3.2 Solution Strategy . . . . . . . . . . . . . . E41

3.3 Numerical Results . . . . . . . . . . . . . . . E42

4 The Optimal Transit Path Problem $\quad$ E45

4.1 The Optimal Control Model . . . . . . . . . . E46

4.2 Results . . . . . . . . . . . . . . . E49

5 Conclusions

E52

References

E53

\section{Introduction}

Consider a general class of optimal control problems where the control functions are assumed piecewise constant and only allowed to take values from a finite discrete set. The aim in such a problem is to find a sequence of discrete control values and a corresponding set of switching times such that a given cost functional is minimized. The difficulty here is that the range set of the control is discrete and hence not convex. Furthermore, choosing the optimal sequence of elements from the control set in an appropriate order is a nonlinear combinatorial optimization problem. Up until recently, even finding the exact switching times for a given control sequence was considered a numerically difficult problem, as the gradient of the 
cost functional with respect to these switching times is known to be discontinuous [7]. However, a novel technique known as the Control Parametrization Enhancing Transform (CPET) was developed in $[4,5,6]$ to overcome this and other difficulties. The CPET is briefly explained in the context of discrete valued optimal control problems. We then consider two application examples arising in defence science.

\section{Problem Formulation and Solution Strategy}

Consider a process described by the following system of nonlinear autonomous differential equations on $\left(0, t_{f}\right]$ :

$$
\dot{\boldsymbol{x}}=\boldsymbol{f}(\boldsymbol{x}, \boldsymbol{u}), \quad \boldsymbol{x}(0)=\boldsymbol{x}_{0},
$$

where $\boldsymbol{x} \in \mathbb{R}^{n}$ is the state vector, $\boldsymbol{u} \in \mathbb{R}^{r}$ is the control vector and $t_{f}$ is the terminal time. The function $\boldsymbol{f}: \mathbb{R}^{n} \times \mathbb{R}^{r} \rightarrow \mathbb{R}^{n}$ is assumed to be continuously differentiable with respect to all its arguments. Consider a piecewise constant function $\boldsymbol{u}:\left[0, t_{f}\right) \rightarrow \boldsymbol{U}$ where $\boldsymbol{U}$ is a finite set in $\mathbb{R}^{r}$. If $\boldsymbol{u}$ has a finite number of discontinuities or switching points, we refer to it as an admissible control. Let $\mathcal{U}$ be the class of all such admissible controls. Note that for a given $\boldsymbol{u} \in \mathcal{U}$, $\boldsymbol{f}(\boldsymbol{x}, \boldsymbol{u})$ may be discontinuous in time at the switching points of $\boldsymbol{u}$. Denote these switching points as $t_{1}, t_{2}, \ldots, t_{N}$ and also define $t_{0}$ and $t_{N+1}$ such that $0=t_{0}<t_{1}<\cdots<t_{N}<t_{N+1}=t_{f}$. The discrete valued optimal control problem is then defined as follows. 
Problem $\left(\boldsymbol{P}_{\mathcal{U}}\right): \quad$ Given the dynamical system (1), find $\boldsymbol{u} \in \mathcal{U}$ such that the cost functional

$$
J_{0}(\boldsymbol{u})=\Phi_{0}\left(\boldsymbol{x}\left(t_{f}\right)\right)+\int_{0}^{t_{f}} \mathcal{L}_{0}(\boldsymbol{x}, \boldsymbol{u}) d t
$$

is minimized subject to the constraints:

$$
\begin{aligned}
J_{i}(\boldsymbol{u})= & \Phi_{i}\left(\boldsymbol{x}\left(t_{f}\right)\right)+\int_{0}^{t_{f}} \mathcal{L}_{i}(\boldsymbol{x}, \boldsymbol{u}) d t \\
& \begin{cases}=0, & i=1,2, \ldots, N_{e}, \\
\geq 0, & i=N_{e}+1, N_{e}+2, \ldots, N_{T} .\end{cases}
\end{aligned}
$$

A control $\boldsymbol{u} \in \mathcal{U}$ is said to be a feasible control if it satisfies the constraints (3). Let $\mathcal{F}(\mathcal{U})$ be the set of all such feasible controls. We assume that $\mathcal{F}(\mathcal{U})$ is non empty, and that Problem $\left(\boldsymbol{P}_{\mathcal{U}}\right)$ admits a solution.

Assumption 1 A solution of $\left(\boldsymbol{P}_{\mathcal{U}}\right)$ has a finite number of switchings.

Suppose that $\boldsymbol{U}=\left\{\boldsymbol{u}^{1}, \boldsymbol{u}^{2}, \ldots, \boldsymbol{u}^{m}\right\}$. We want to determine an optimal sequence in which elements from $\boldsymbol{U}$ occur as well as the switching times corresponding to this sequence. Let us initially solve the problem for a fixed number of $N$ switchings. Construct a set $\left\{\boldsymbol{b}_{i}\right\}_{i=1}^{M}, M=(N+1) m$, where $\boldsymbol{b}_{i}=\boldsymbol{u}^{((i-1) \bmod m)+1}, i=$ $1,2, \ldots, M$. Then any $\boldsymbol{u} \in \mathcal{U}$ which has $N$ switchings can be written as

$$
\boldsymbol{u}(t)=\boldsymbol{b}_{i}, \quad t \in\left[\tau_{i-1}, \tau_{i}\right),
$$

where the $\tau_{i} \in\left[0, t_{f}\right]$ satisfy $0=\tau_{0} \leq \tau_{1} \leq \tau_{2} \leq \cdots \leq \tau_{M-1} \leq \tau_{M}=$ $t_{f}$. Note that $\boldsymbol{u}$ is now determined only by $\tau_{i}, i=1,2, \ldots, M-1$. We then solve Problem $\left(\boldsymbol{P}_{\mathcal{U}}^{N}\right)$, which is to determine a set of $\tau_{i}$, 
$i=1,2, \ldots, M-1$, such that the resulting $\boldsymbol{u}_{N}$ minimizes (2) subject to $(3)$.

Note that a solution of Problem $\left(\boldsymbol{P}_{\mathcal{U}}^{N}\right)$ is only optimal with respect to a fixed number of switchings $N$. The question of actually finding the optimal number of switchings has not been solved for the general class of problems considered here. In addition, the approach outlined here cannot guarantee that we obtain the globally optimal sequence of controls. In some practical problems, such as the application examples given later, this is not particularly important, but in others, it is. A reasonable heuristic approach is to start with a fixed $N$ and solve the Problem $\left(\boldsymbol{P}_{\mathcal{U}}^{N}\right)$. We then increase $N$ and solve $\left(\boldsymbol{P}_{\mathcal{U}}^{N}\right)$ again. If there is no decrease in the optimal cost, we assume the previous value of $N$ to yield the optimal number of switchings. Otherwise we increase $N$ further. Under Assumption 1, we will not have to increase $N$ indefinitely.

For a given set $\left\{\tau_{i}\right\}_{i=0}^{M}$, the right hand side of the dynamics may be discontinuous as a function of $t$ at $\tau_{i}, i=1,2, \ldots, M-1$. Because of the manner in which they appear in the problem, there are a number of numerical difficulties when trying to calculate the optimal $\tau_{1}, \tau_{2}, \ldots, \tau_{M-1}$ in a conventional way [5]. To overcome these, we employ the Control Parametrization Enhancing Technique (CPET) developed in $[4,5,6]$.

We introduce the new time variable $s \in[0, M]$. Let $\mathcal{V}$ denote the class of non-negative piecewise constant scalar functions defined on $[0, M)$ with fixed switching points located at $\{1,2,3, \ldots, M-1\}$. The transformation (СРЕT) from $t \in\left[0, t_{f}\right]$ to $s \in[0, M]$ is defined by

$$
\frac{d t}{d s}=v(s), \quad t(0)=0,
$$

where the scalar function $v \in \mathcal{V}, v(s)=\tau_{i}-\tau_{i-1}$ for $s \in[i-1, i)$, 
is called the enhancing control. We also impose the constraint

$$
\int_{0}^{M} v(s) d s=t_{f}
$$

Under the CPET, each admissible control $\boldsymbol{u}(t) \in \mathcal{U}$ given by (4) is transformed into $\boldsymbol{\mu}$, where $\boldsymbol{\mu}(s)=\boldsymbol{b}_{i}, s \in[i-1, i), i=1,2, \ldots, M$. Whereas the fixed function $\boldsymbol{\mu}$ contains many artificial switchings to allow all the possible orderings of the sequence of discrete control values to be considered when solving the transformed problem, these do not have any bearing on the original problem, since if $v(s)=0$ on $[i-1, i)$, then $\tau_{i-1}=\tau_{i}$ and there is no switching in the original time scale.

We now transform Problem $\left(\boldsymbol{P}_{\mathcal{U}}^{N}\right)$. Note that by substituting (5) into (1), (2) and (3), we obtain the following equivalent problem.

Problem $\left(\widetilde{\boldsymbol{P}_{\mathcal{U}}^{N}}\right)$ : $\quad$ Subject to the system equations and initial condition

$$
\frac{d \boldsymbol{x}}{d s}=v(s) \boldsymbol{f}(\boldsymbol{x}(s), \boldsymbol{\mu}(s)), \quad \boldsymbol{x}(0)=\boldsymbol{x}_{0},
$$

and the constraints

$$
\begin{aligned}
\hat{J}_{i}(v(s))= & \Phi_{i}(\boldsymbol{x}(M))+\int_{0}^{M} v(s) \mathcal{L}_{i}(\boldsymbol{x}(s), \boldsymbol{\mu}(s)) d s \\
& \begin{cases}=0, & i=1,2, \ldots, N_{e}, \\
\geq 0, & i=N_{e}+1, \ldots, N_{T},\end{cases}
\end{aligned}
$$

find a $v \in \mathcal{V}$ satisfying (6) such that the cost functional

$$
\hat{J}_{0}(v(s))=\Phi_{0}(\boldsymbol{x}(M))+\int_{0}^{M} v(s) \mathcal{L}_{0}(\boldsymbol{x}(s), \boldsymbol{\mu}(s)) d s
$$

is minimized. We denote the feasible subset of $\mathcal{V}$ as $\mathcal{F}(\mathcal{V})$ where feasibility relates to (6) and (8). Note that, rather than finding a 
set of feasible switchings $\tau_{1}, \tau_{2}, \ldots, \tau_{M-1}$ to minimize $(2),\left(\widetilde{\boldsymbol{P}_{\mathcal{U}}^{N}}\right)$ seeks to find $v(\cdot) \in \mathcal{F}(\mathcal{V})$ to minimize (9). All switching points of the original control are mapped onto the set of integers in chronological order. Piecewise integration can now be performed easily since all points of discontinuity in the $s$-domain are known and fixed. Moreover, $v(\cdot) \in \mathcal{V}$ is a piecewise constant function and hence Problem $\left(\widetilde{\boldsymbol{P}_{\mathcal{U}}^{N}}\right)$ is readily solvable by the optimal control software MISER3.2 [3]. For any $\hat{s} \in[0, M]$, the corresponding $\hat{t} \in\left[0, t_{f}\right]$ is given by $\hat{t}=t(\hat{s})=\int_{0}^{\hat{s}} v(s) d s$, so the state and the control histories of the original problem defined on $\left[0, t_{f}\right)$ can be reconstructed easily.

\section{The Optimal Battery Recharge Problem}

Consider a submarine with three diesel generators, each of which is operated in either ordinary mode or in supercharged mode. The aim of a battery recharge plan is to optimally choose a strategy of running the generators in a number of possible modes and for varying lengths of time over a given period in order to maintain charge in the battery bank. We need to decide how many recharge intervals to employ, how many engines to run during each recharge interval and what mode these should be run in, while allowing the submarine to travel a fixed distance.

The primary objective is the minimization of the total recharge time in order to minimize exposure of the vessel to the enemy. The objective function should also reflect the fact that the various modes of operation result in different levels of exposure. Finally, it should take into account the need to maintain a reasonable charge level in the battery banks. 


\subsection{The Optimal Control Model}

The optimal control model comprises of the battery recharge dynamics, the objective functional and a set of constraints. We start with a time horizon $\left[0, t_{f}\right]$ and let $C(t)$ denote the charge state of the battery pack at time $t$. The speed of the vessel is denoted by the control function $s(t)$. We assume that the speed remains constant over each individual discharge or recharge interval. Note that $s(t)$ is not a discrete valued control, since it is allowed to take on any value between the bounds given below. We define two discrete valued control functions whose values depend on how many engines are running at any given time and the mode of operation:

- $u_{1}(t)$ indicates what fraction of full mechanical power is being produced by the engines at time $t$;

- $u_{2}(t)$ gives an indication of the level of noise produced by the combination and mode of operation of generators operating at time $t$.

The values of $u_{1}$ and $u_{2}$ for the various possible operational modes are given in Table 1 . For the $u_{2}$ values, $u_{\text {noise }}$ is a parameter which represents the noise power output of a single engine running in normal mode. The subsequent values of $u_{2}$ then assume that noise power increases linearly with the number of engines used and by $50 \%$ when engines are run in supercharged instead of normal mode. These values would be calibrated as required for an actual submarine. Note that there is a total of only 7 operational modes. The charge dynamics are given by $\dot{C}(t)=R\left(t, u_{1}(t)\right)-D\left(t, s(t), u_{1}(t)\right)$, $C(0)=C_{\text {start }}$, where $R\left(t, u_{1}(t)\right)=20000 u_{1}(t) /[C(t)+100]$ and

$$
D(t, s(t))= \begin{cases}s^{2}(t) / 120+8, & \text { if } u_{1}(t) \neq 0, \\ s^{2}(t) / 80+5, & \text { if } u_{1}(t)=0 .\end{cases}
$$




\begin{tabular}{|lcc|}
\hline Operational Mode (engines) & Value of $u_{1}$ & Value of $u_{2}$ \\
\hline Discharging (none running) & 0 & 0 \\
Recharging (one normal) & 0.2564 & $u_{\text {noise }}$ \\
Recharging (one supercharged) & 0.3333 & $1.5 \times u_{\text {noise }}$ \\
Recharging (two normal) & 0.5128 & $2.0 \times u_{\text {noise }}$ \\
Recharging (two supercharged) & 0.6666 & $3.0 \times u_{\text {noise }}$ \\
Recharging (three normal) & 0.7692 & $3.0 \times u_{\text {noise }}$ \\
Recharging (three supercharged) & 1.0 & $4.5 \times u_{\text {noise }}$ \\
\hline
\end{tabular}

TABLE 1: Values of $u_{1}$ and $u_{2}$.

Subject to these dynamics, we then want to minimize

$$
\int_{0}^{t_{f}}\left\{\alpha(100-C(t))^{2}+\beta \ln \left(u_{2}(t)+1\right)\right\} d t .
$$

The first term in this objective measures the deviation of the charge state from the full level, while the second term measures the exposure of the vessel due to recharging. The weights $\alpha$ and $\beta$ are chosen appropriately. We impose a number of constraints on the problem. The speed of the vessel is restricted to between 5 and $60 \mathrm{~km} / \mathrm{h}$, that is, $s(t) \in[5,60]$ for all $t \in\left[0, t_{f}\right]$. The submarine must cover a distance of $L \mathrm{kms}$, that is, $\int_{0}^{t_{f}} s(t)=L$. We place bounds on the charge state of the battery:

$$
20 \leq C(t) \leq 100, \text { for all } t \in\left[0, t_{f}\right] .
$$

Finally, we ensure that the submarine ends the journey with a sufficiently high charge state, that is, $C\left(t_{f}\right) \geq C_{\min }$.

\subsection{Solution Strategy}

While this model is an example of a discrete valued optimal control problem, there are two additional features which we did not consider 
in Problem $\left(\boldsymbol{P}_{\mathcal{U}}\right)$. First, in addition to the discrete valued control functions, we have the control $s(t)$ which can take any value in the given interval. Assuming that this additional control is a piecewise constant function which switches at the same time points as the discrete valued controls, there are no difficulties to incorporate it. Even if different switching points for $s(t)$ are desired, this is easily achieved by a simple increase in the number of allowable switchings. The second additional feature is the existence of the all time state constraints (10). They are dealt with using the method described in [2], already incorporated into the MISER3.2 software.

\subsection{Numerical Results}

We consider the following parameter settings: $t_{f}=24, C_{\text {start }}=$ $100, u_{\text {noise }}=10, L=840$ and $C_{\min }=20$. We assume that any type of recharge interval can occur a maximum of 10 times over the time horizon and calculate optimal solutions for various values of $\alpha$ and $\beta$. For example, $\alpha=0.001$ and $\beta=1$ results in the solution shown in Figures 1 and 2. The values along the vertical axis in Figure 2(b) are interpreted as follows: $0 \equiv$ no engine running; $1 \equiv$ one engine (normal mode); $2 \equiv$ one engine (supercharged); $3 \equiv$ two engines (normal mode) 4 E two engines (supercharged) $; 5 \equiv$ three engines (normal mode); and $6 \equiv$ three engines (supercharged). Lower values of $\beta$ result in extended periods of recharging which is unlikely to be acceptable in practise. Much larger values of $\beta$ yield solutions where the charge state is repeatedly driven to the lower bound, which is also not acceptable. Note that, for nearly all of the cases when $\beta>1$, recharging is carried out with all three engines running in supercharged mode. Also, the final charge state is often driven down a long way. This may be undesirable for a practical implementation, as a low charge state reduces manoeuvrability and combat readiness. However, raising the value of $C_{\min }$ does result in 


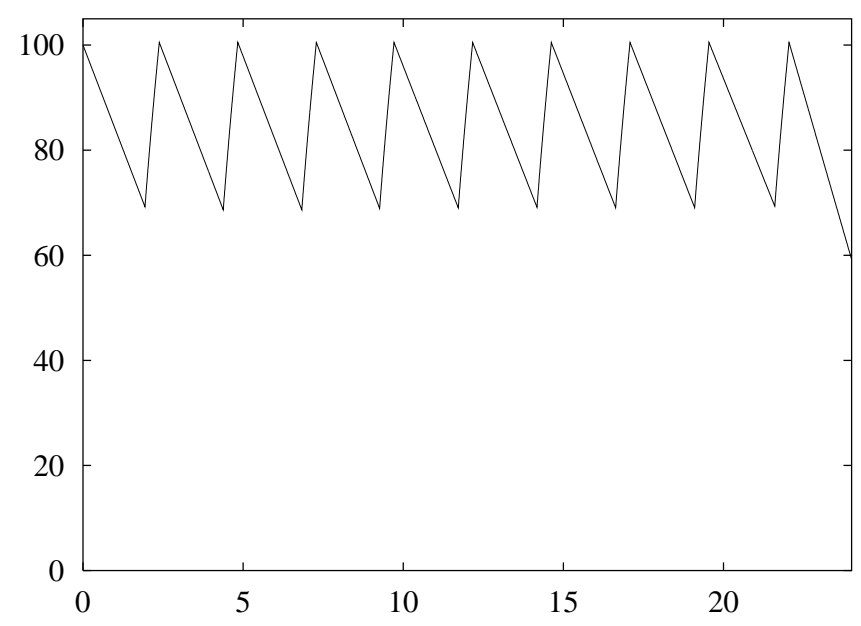

Figure 1: Optimal Capacity as a function of time: $\alpha=0.001$, $\beta=1$.

a more acceptable solution.

The fact that recharging is almost exclusively carried out with all engines running in supercharged mode is a reflection of how we modelled the noise term in the objective functional, including our particular choice of the $u_{\text {noise }}$ value. The reasoning for choosing $u_{\text {noise }}=10$ is that it reflects the operational reality that there is a significant difference between no recharging $\left(u_{2}=0\right)$ and recharging with even just one engine running in normal mode $\left(u_{2}=1\right)$, particularly when the added danger of being surfaced is taken into account. Consequently, there is then a relatively small difference in the 'noise' produced when one engine is running as compared to the case of all three engines running. To then minimize the total recharge time, the optimal solution consequently chooses to recharge as quickly as possible, that is, with as many engines as possible, all 
(a)

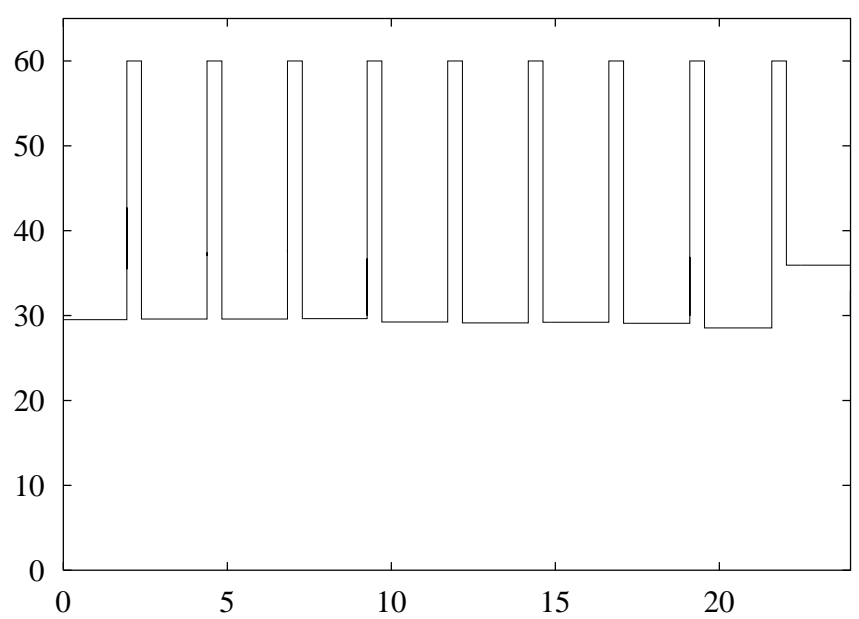

(b)

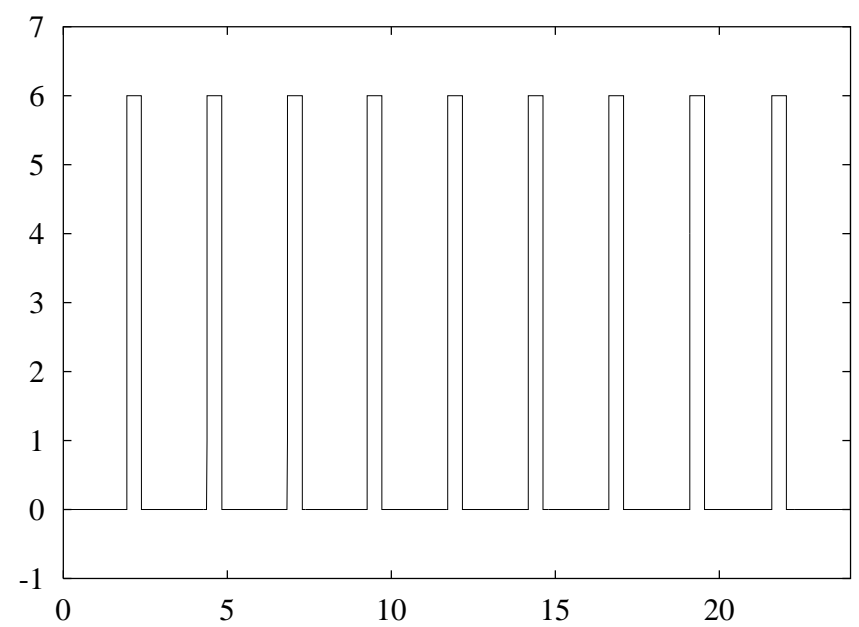

Figure 2: Optimal speed profile, (a), and optimal engine management, (b), over time: $\alpha=0.001, \beta=1$. 
in supercharged mode. By modifying this aspect of the objective functional, we have been able to obtain solutions which are qualitatively very different. However, it is felt that these modifications make the model too unrealistic.

Finally, note that it is possible to modify the model in a number of practical ways. For example, by adding an appropriately weighted term to the objective functional, we discourage recharging over certain time intervals if there is more danger in the submarine surfacing during those periods.

\section{The Optimal Transit Path Problem}

Here we consider the problem of determining a safe path which a submarine should follow through a field of sonar sensors. Each of the sensors detects the presence of the submarine with a probability which is a given function of the distance between the two and of the speed of the submarine. This function is not a simple analytical expression, but depends upon a range of factors, including the characteristics of the ocean floor and ocean surface, depths of the sensor and the submarine, and the temperature and salinity of the water. See [1] and the references cited therein for more details. In this paper, we use probability of detection functions reported in [1], constructed under the assumptions that the geographic location and environmental conditions are known and that the submarine remains at a constant depth. Each of the functions are constructed for a particular constant vessel speed. While there are still further factors influencing the probability of detection, the functions from [1] contain sufficient detail to test the feasibility of the proposed method.

The objective then is to find a transit path between two fixed 
positions in the region of interest which will minimize the overall probability of detection while still satisfying a maximum time constraint.

Previous work in this area [1] has concentrated on creating a network structure over the sensor field and then using network optimization techniques and heuristic search procedures to find optimal paths in terms of node and arc sets. Here, we propose an optimal control formulation with a simple dynamical system to describe the movement of the vessel. The resulting problem is a discrete valued optimal control problem.

\subsection{The Optimal Control Model}

The region of interest is modelled by the Cartesian plane with coordinates $(x, y)$ indicating the latitudinal and longitudinal distance from the origin in kilometres, where the origin is assumed to be the starting point of the journey. We let $(x(t), y(t))$ represent the (point) location of the submarine at time $t$, assumed to be governed by

$$
\dot{x}(t)=s(t) \cos \theta(t), \quad \dot{y}(t)=s(t) \sin \theta(t), \quad x(0)=y(0)=0,
$$

where $\theta(t)$ represents the heading angle of the vessel in radians and $s(t)$ is the speed of the vessel in $\mathrm{km} / \mathrm{h}$. Note that $\theta$ and $s$ are control functions here, subject to the constraints

$$
0 \leq \theta(t) \leq 2 \pi, \quad s(t) \in\{8,14\}, \quad \text { for all } t \in\left[0, t_{f}\right),
$$

where $t_{f}$ is the terminal time of the journey. Clearly, $s(t)$ is a discrete valued control function. Physically, a discrete valued vessel speed is obviously not realistic. However, at the planning level, it is natural for human operators to think of the journey in terms of 
discrete stages with each stage corresponding to a constant speed section of the journey.

Suppose that a total of $n_{s}$ sensors are located at positions $\left(x_{i}, y_{i}\right)$, $i=1,2, \ldots, n_{s}$, in the field. We assume for simplicity that these positions remain fixed during the journey and that the sonars are all of the same type with the same detection capabilities. Either of these assumptions can easily be relaxed in the model and solution method described below. At any instant of time, the distance of the submarine from each sensor is

$$
r_{i}(t)=\sqrt{\left(x(t)-x_{i}\right)^{2}+\left(y(t)-y_{i}\right)^{2}}, \quad i=1, \ldots, n_{s} .
$$

For each given vessel speed $s$, a probability of detection profile, $p(r, s)$ is constructed as a function of the physical distance $r(t)$. For the purposes of our model, we use a cubic spline interpolation of the data in [1], as illustrated in Figure 3. Note that a separate profile is required for each vessel speed.

Assuming that the sensors operate independently, the instantaneous probability of the vessel being detected is then

$$
\operatorname{Pr}(x(t), y(t), s(t))=1-\prod_{i=1}^{n_{s}}\left[1-p_{i}\left(r_{i}(t), s(t)\right)\right],
$$

where $p_{i}\left(r_{i}(t), s(t)\right), i=1, \ldots, n_{s}$, are the probabilities of being detected by the individual sensors.

Our aim is to minimize the cumulative probability of being detected over the entire journey. This is equivalent to minimizing the objective functional

$$
g\left(\theta, s, t_{f}\right)=\int_{0}^{t_{f}} \operatorname{Pr}(x(t), y(t), s(t)) d t .
$$




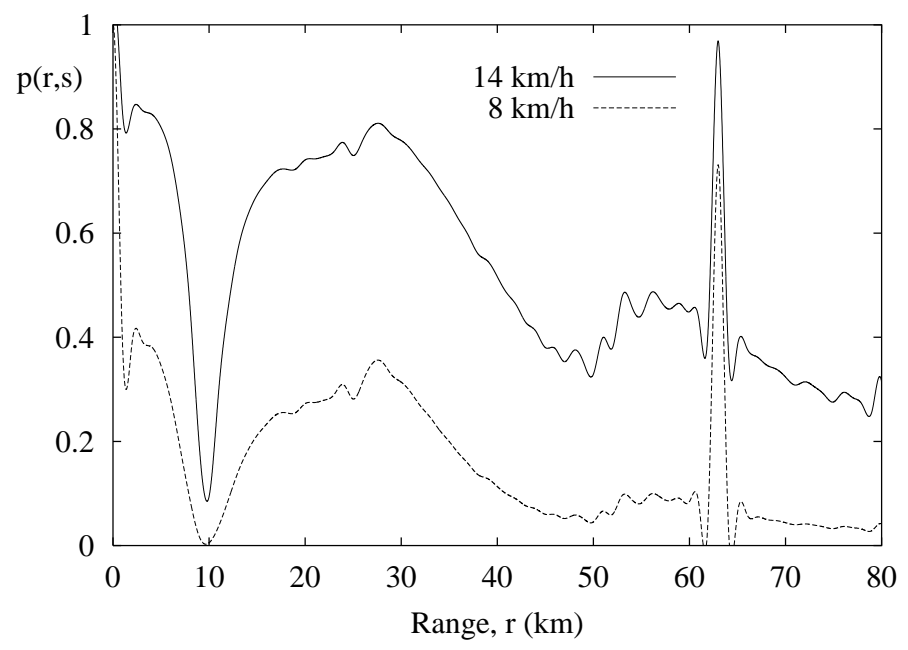

Figure 3: Probability of detection curves corresponding to speeds of $14 \mathrm{~km} / \mathrm{h}$ and $8 \mathrm{~km} / \mathrm{h}$, interpolated from the data in [1].

Note that the submarine must arrive at its intended destination, so we have the terminal state constraints

$$
x\left(t_{f}\right)=x_{f}, \quad y\left(t_{f}\right)=y_{f} .
$$

The terminal time, $t_{f}$, is variable in this problem, with

$$
t_{f} \leq T_{\max } .
$$

In summary, then, the optimal control model of the submarine transit path problem is: Find a terminal time $t_{f}$ (satisfying (15)), and control functions $\theta(t)$ and $s(t)$ (satisfying (12)) such that the objective functional (13) is minimized subject to the vessel dynamics (11) and the constraints (14).

Once again, we are dealing with a discrete valued optimal control problem. As for the optimal battery recharge problem, here we have 
a control function, $\theta(t)$, which is not discrete valued. However, as before, with some mild assumptions stated below, this can also be easily incorporated into the CPET.

We need to set a limit to the number of course/speed switchings to be allowed. Note that the heading angle control function, $\theta(t)$, is modelled as a piecewise constant function, which is natural, given that the heading angle ought to remain constant between course changes. For simplicity, we assume that the switching times for the course changes coincide with switching times for the speed changes. This may appear to be restrictive, but since we allow for only one of the controls to change values at a particular switching time, full generality of the control is preserved. A submarine commander is unlikely to implement a solution which involves an excessive number of course/speed changes, so we assume a maximum number of switchings $N$.

\section{$4.2 \quad$ Results}

We have restricted our examples to sonar fields with $n_{s}=4$ sensors. We choose $x_{0}=0, y_{0}=0, x_{f}=80$ and $y_{f}=80$. For our simulations, the sonar sensors are located at

$$
\{(20,60),(40,60),(60,60),(70,10)\}
$$

relative to the starting point of the journey. We assume that the journey is to be completed within a day, so $T_{\max }=24$. Furthermore, we allow a maximum of $N=72$ course or speed changes. We use the probability of detection curves shown in Figure 3. The solution is illustrated in Figure 4, where the transit path is indicated by the thin white line. Note that the thick circular white lines indicate regions where the probability of detection is extremely high and 
these correspond to the peaks of the curves in Figure 3 at approximately $63 \mathrm{~km}$. A feature of the solution not immediately obvious from the figure is that the speed of the vessel changes according to the level of danger present in the various sections of the paths. It can be seen the transit path stays close to low danger areas as long as it is not forced into a major detour. If this is not possible, the path does end up crossing high probability of detection regions a number of times. When these high danger areas are traversed, contrary to what one might expect, the optimal solution is to do so at the high speed. In this manner, the total amount of time spent in the high danger regions is reduced, and thus the cumulative effect of exposure is minimized. This is clearly a direct reflection of the objective function used in our model, which implicitly seeks to minimize the total transit time. Since even short time periods spent in high danger areas are highly undesirable, the chosen objective function may require modifications to more accurately reflect the needs of vessel operators.

The time duration for the optimal path of the above problem is 12 hours and 53 minutes, less than the allowed 24 hours, that is, the total time constraint is not active for this solution. To see the behaviour in the case of when the time constraint is active, we have a simulation where $T_{\max }=10$. The resulting path takes a more direct route, going almost diagonally from the start to the finish point. The duration and number of high speed intervals is also greater in this case, as expected.

Our numerical studies have shown that, for some sensor patterns and probability of detection profiles, the solutions obtained are highly dependent on the initial guesses provided to the software. In other words, the solutions we have obtained here are only local optima. It is quite easy to see the reason for this in the probability of detection pattern resulting from the sensor field. There are 


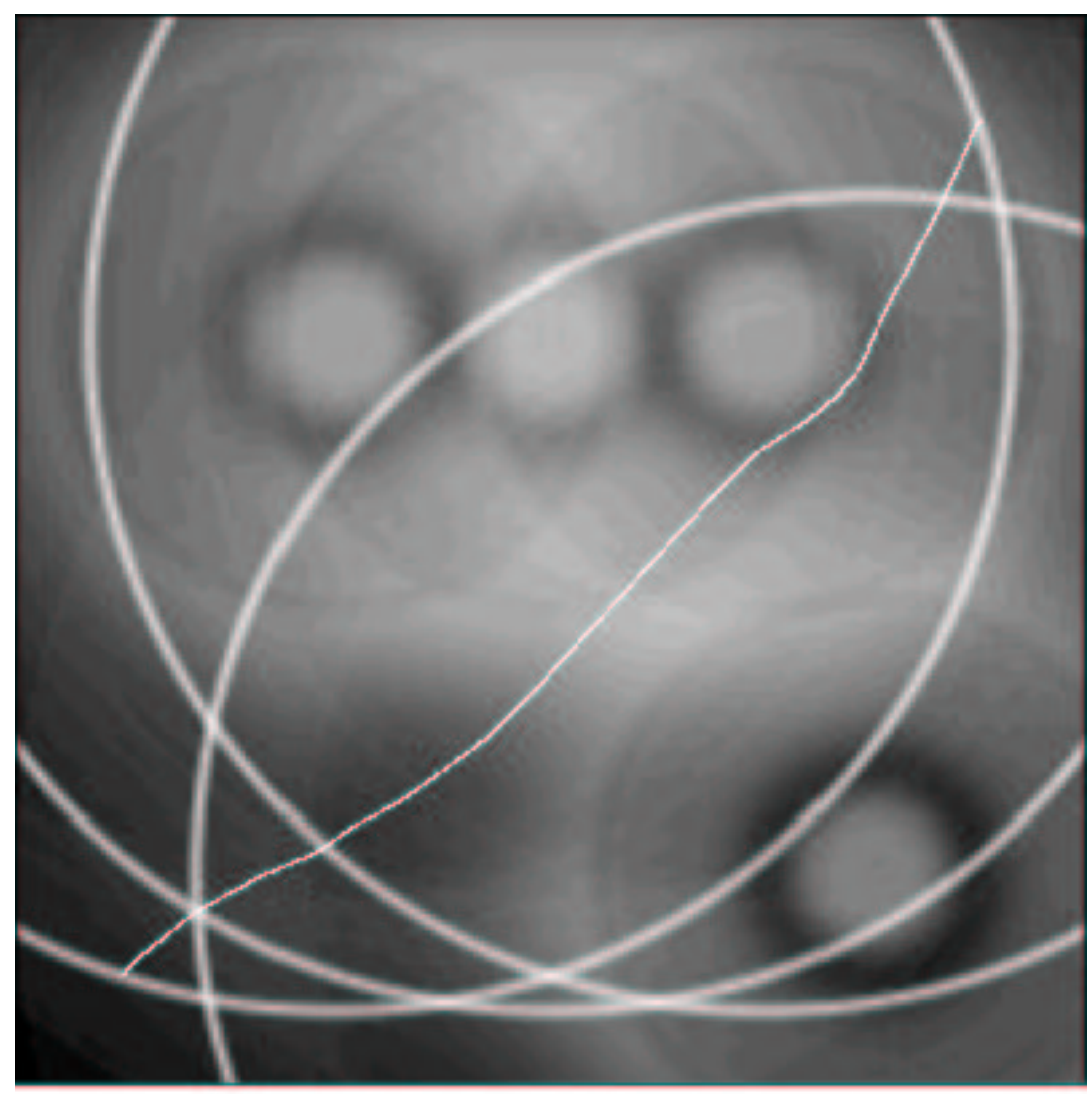

$3 \%$

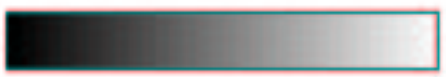

$95 \%$

Probability of Detection

Figure 4: Optimal transit path is the thin white line through four detectors at the middle of the circles. 
many possible paths to the destination and once the basic movements are determined (as a consequence of the initial guess), it is unlikely that they are altered again during the optimization to yield a qualitatively different path. Despite this drawback, the optimal control approach to the transit path problem is clearly able to generate good locally optimal solutions. In addition, it is quite easy to incorporate more complex path constraints and dynamics, rather than static, sensor patterns. The authors are currently developing global optimization strategies for this problem.

\section{Conclusions}

We have presented two practical problems arising in defence science, both modelled as optimal control problems. In each case, some of the controls are discrete valued. A brief review of a general class of discrete valued optimal controls was presented, along with a solution strategy via the Control Parametrization Enhancing Transform. Numerical results are given for both application examples.

Acknowledgements: We gratefully acknowledge financial support from the Maritime Operations Division in the Defence Science and Technology Organization for this research work. In particular, we are thankful to Robert O'Dowd for giving us the opportunity to work on these applications and to Christina Hallam for her discussions on the transit path problem and for providing some of the figures in this paper. 


\section{References}

[1] C.L. Hallam, "Hierarchical path generation: An application to submarine transit paths", Honours Dissertation, Murdoch University, Western Australia, 1997. E45, E46, E47, E48

[2] L.S. Jennings and K.L. Teo, "A computational algorithm for functional inequality constrained optimization problems", Automatica 26 (1990) 371-375. E42

[3] L.S. Jennings, K.L. Teo, M.E. Fisher and C.J. Goh, "MISER3 version 2, optimal control software, theory and user manual", http://cado . maths. uwa.edu.au/miser/manual. html, Department of Mathematics, University of Western Australia, 1997. E39

[4] H.W.J. Lee, K.L. Teo, L.S. Jennings and V. Rehbock, "Control parametrization enhancing technique for time optimal control problems", Dynamical Systems and Applications 6 (2) (1997) 243-261. E35, E37

[5] H.W.J. Lee, K.L. Teo, V. Rehbock and L.S. Jennings, "Control parametrization enhancing technique for discrete-valued control problems", Automatica 35 (8) (1999) 1401-1407. E35, E37

[6] V. Rehbock, K.L. Teo, L.S. Jennings and H.W.J. Lee, "A survey of the control parametrization and control parametrization enhancing methods for constrained optimal control problems", in Progress in Optimization: Contributions from Australasia (ed. A. Eberhard et al.), (Kluwer Academic Publishers, 1999) 247-275. E35, E37 
[7] K.L. Teo, C.J. Goh and K.H. Wong, A Unified Computational Approach to Optimal Control Problems (Longman Scientific and Technical, Essex, 1991). E35 